\title{
EDITORIAL
}

\section{Prognostic importance of right ventricular dysfunction}

\author{
R A Bleasdale, M P Frenneaux
}

Heart 2002;88:323-324

\begin{abstract}
Although left ventricular ejection fraction is a powerful predictor of mortality following a myocardial infarction, in certain situations right ventricular ejection fraction may provide additional prognostic information
\end{abstract}

W hen considering the entire range of left ventricular ejection fractions (LVEF) after a myocardial infarction, this parameter is a powerful predictor of mortality. ${ }^{1}$ However extended follow up studies have shown that prolonged survival occurs in some patients with congestive heart failure (CHF) who have adverse conventional prognostic markers, including very low LVEF. ${ }^{2}$ In patients with LVEF < 20\%, the absolute value has little prognostic significance but peak oxygen consumption (peak $\dot{\mathrm{V}}_{2}$ ) is predictive. There is increasing evidence that right ventricular ejection fraction (RVEF) is also a powerful predictor of mortality in this subgroup of patients - perhaps the most powerful predictor.

In patients with CHF caused by dilated cardiomyopathy (DCM), reduced RVEF assessed using gated heart pool scanning $(<38 \%)$ has been shown to be associated with increased mortality in patients with LVEF $<30 \%$ but not in those patients with higher LVEFs. ${ }^{1}$ Similarly, in patients with ischaemic heart disease (IHD), RVEF is prognostically important in those patients with LVEF $<40 \%{ }^{3}$ In a study undertaken in a cardiac transplant waiting list population (of mixed aetiology), patients were stratified according to RVEF greater or less than $24 \%$. RVEF was shown to be an independent predictor of survival in a multivariate model that included LVEF. One year survival in the two groups was 44\% and 93\%, respectively. ${ }^{4}$ Juilliere and colleagues confirmed that LVEF and RVEF were both independent predictors of survival in a multivariate model. ${ }^{5}$ In another study of patients with mean (SD) LVEF $22(7) \%$, resting RVEF (> 35\%) was shown to be the most powerful independent predictor of survival in a multivariate model which included the other conventional prognostic markers (LVEF, peak $\dot{\mathrm{V}}_{\mathrm{O}_{2}}$, peak percentage age and sex adjusted $\dot{\mathrm{V}}_{\mathrm{O}_{2}}$, left ventricular end diastolic volume index, left ventricular end systolic volume index, and cardiac index). ${ }^{6}$

The presence of right ventricular involvement also has a major effect on short term outcome in patients with acute inferior myocardial infarction (odds ratio for in hospital mortality 3.2). ${ }^{7}$ Successful primary angioplasty promptly normalises RVEF in this setting and is associated with improved in-hospital mortality compared with patients in whom primary angioplasty is unsuccessful $(2 \%$ v $58 \%))^{8}$

\section{DETERMINANTS OF RVEF IN PATIENTS WITH CHF}

RVEF is determined by intrinsic right ventricular contractile function, and by right ventricular preload and afterload. Because DCM generally affects both ventricles, RVEF tends to be lower for any given LVEF in patients with DCM versus those with IHD. ${ }^{9}$ It is important to note that the function of the left ventricle can influence that of the right ventricle in two important ways. Firstly, the interventricular septum is shared by both ventricles, and contributes substantially to right ventricular contractile function. ${ }^{10}$ Secondly, RVEF is influenced by the afterload against which it has to eject (total pulmonary resistance), which is also greatly affected by left ventricular end diastolic pressure (LVEDP) as well as by pulmonary vascular resistance. ${ }^{11}$ Therefore RVEF was negatively associated with raised mean pulmonary artery pressure and with raised right ventricular end diastolic pressure (RVEDP), with the combination being additive in predicting RVEF. $^{11}$

For these reasons, in patients with acute anterior myocardial infarction, in whom the infarct is limited to the anterior wall of the left ventricle and the upper septum, right ventricular ejection fraction is depressed at day 2, but recovers by day 10 despite persistent impairment of LVEF. ${ }^{12}$

\section{WHY DOES RVEF DETERMINE OUTCOME?}

The answer to this is unknown. It is certainly possible that the presence of additional right ventricular involvement may increase the substrate for malignant ventricular arrhythmias. However, the link between RVEF and mortality appears also to affect deaths caused by intractable $\mathrm{CHF}^{7}$ This is therefore unlikely to be the sole explanation.

It was noted earlier that the combination of increased RVEDP and increased mean pulmonary artery pressure potently predict reduced RVEF. Such patients are almost certain to exhibit pronounced diastolic ventricular interaction (DVI). ${ }^{13}$ In the presence of an enlarged heart, particularly when associated with right ventricular systolic hypertension, the pericardium and other surrounding structures (including the lungs) become stretched, and pericardial pressure (normally close to zero) becomes greatly elevated. Because the right ventricle is relatively thin

Abbreviations: CHF, congestive heart failure; DCM, dilated cardiomyopathy; DVI, diastolic ventricular interaction; IHD, ischaemic heart disease; LVEDP, left ventricular end diastolic pressure; LVEF, left ventricular ejection fraction; RVEDP, right ventricular end diastolic pressure; RVEF, right ventricular ejection fraction; $\dot{V}_{\mathrm{O}_{2}}$ oxygen consumption 
walled, and unable to maintain a significant transmural pressure gradient, RVEDP usually provides a very close estimate of pericardial pressure. In such patients, external constraint from the pericardium and right ventricle (via the shared septum) restrains the filling of the left ventricle. Increases in LVEDP such as during exercise fail to cause the expected increase in left ventricular end diastolic volume and therefore (via the Starling mechanism) stroke volume, resulting in a greatly reduced ability to increase cardiac output on exercise. ${ }^{14}$ Consistent with this, RVEF is much more powerful than LVEF as a predictor of exercise capacity in $\mathrm{CHF} .{ }^{15} \mathrm{CHF}$ patients with DVI may be recognised non-invasively by the presence of a restrictive transmitral Doppler flow profile, ${ }^{16}$ itself a powerful adverse prognostic marker. ${ }^{17}$ The presence of DVI appears also to be associated with baroreflex abnormalities ${ }^{18}$ and with greater adverse neurohumoral activation. ${ }^{19}$ This is likely to be caused by the specific impact of DVI on stretch sensitive receptors in the left ventricle as well as by reduced cardiac output. Baroreflex dysfunction and neurohumoral activation are causally associated with both sudden death and disease progression. ${ }^{2021}$ Thus, while speculative, DVI may provide a plausible explanation for the effects of RVEF on both exercise capacity and survival in patients with CHF.

\section{ASSESSMENT OF RIGHT VENTRICULAR FUNCTION}

The assessment of RV function has historically been very difficult. Standard transthoracic echocardiography has been of limited use and so the majority of the literature is based on radionuclide ventriculography. Recent developments in echocardiographic technology, in particular the development of tissue Doppler imaging, have changed this situation. Long axis velocities of the right ventricle (free wall and tricuspid annulus) have been shown to be accurate and reproducible measures of right ventricular systolic function. ${ }^{22}$ Patients with LVEF $<30 \%$ on stable maximal medical treatment might therefore appropriately undergo evaluation of right ventricular systolic function using this technique or using radionuclide ventriculography.

\section{CONCLUSION}

RVEF provides powerful prognostic information in patients with CHF, particularly in those with very low LVEF. Reduced RVEF may be a consequence of both intrinsic right ventricular disease and of increased right ventricular afterload. The mechanisms likely to be responsible for the prognostic impact of reduced RVEF are uncertain, but have been discussed.

\section{ACKNOWLEDGEMENTS}

Professor Frenneaux and Dr Bleasdale are supported by the British Heart Foundation.

\section{Authors' affiliations}

R A Bleasdale, M P Frenneaux, Department of Cardiology, Wales Heart Research Institute, University of Wales College of Medicine, Cardiff, UK

\section{REFERENCES}

1 Shah PK, Maddahi J, Staniloff HM, et al. Variable spectrum and prognostic implications of left and right ventricular ejection fractions in patients with and without clinical heart failure after acute myocardial infarction. Am J Cardiol 1986;58:387-93.

2 Niebaver J, Clark AL, Anker SD, et al. Three year mortality in heart failure patients with very low left ventricular ejection fractions. Int J Cardiol 1999:70:245-7

3 Polak JF, Holman BL, Wynne J, et al. Right ventricular ejection fraction: an indicator of increased mortality in patients with congestive heart failure associated with coronary artery disease. J Am Coll Cardiol 1983;2:217-24.

4 Gavazzi A, Berzuini C, Campana C, et al. Value of right ventricular ejection fraction in predicting short-term prognosis of patients with severe chronic heart failure. J. Heart Lung Transplant 1997; 16:774-85.

5 Juilliere $Y$, Barbier $G$, Feldmann L, et al. Additional predictive value of both left and right ventricular ejection fractions on long-term survival in idiopathic dilated cardiomyopathy. Eur Heart J 1997; 18:276-80.

6 Di Salvo TG, Mathier M, Semigran M, et al. Preserved right ventricular ejection fraction predicts exercise capacity and survival in advanced heart failure. J Am Coll Cardiol 1995;25:1 143-53.

7 Mehta SR, Eikelboom JW, Natarajan MK, et al. Impact of right ventricular involvement on mortality and morbidity in patients with inferior myocardial infarction. J Am Coll Cardiol 2001;37:37-43.

8 Bowers TR, O'Neill WW, Grines C, et al. Effect of reperfusion on biventricular function and survival after right ventricular infarction. $N$ Engl J Med 1998;338:933-40.

9 La Vecchia L, Zanolla L, Varotto L, et al. Reduced right ventricular ejection fraction as a marker for idiopathic dilated cardiomyopathy compared with ischemic left ventricular dysfunction. Am Heart J $2001 ; 142: 181-9$

10 Nakamura S, Iwasaka T, Kimura Y, et al. Right ventricular ejection fraction during exercise in patients with recent myocardial infarction: effect of the interventricular septum. Am Heart J 1994;127:49-55.

11 Korr KS, Gandsman EJ, Winkler ML, et al. Hemodynamic correlates of right ventricular ejection fraction measured with gated radionuclide angiography. Am J Cardiol 1982;49:71-7.

12 Marmor A, Geltman EM, Biello DR, et al. Functional response of the right ventricle to myocardial infarction: dependence of the site of left ventricular infarction. Circulation 1981;64:1005-11.

13 Atherton JJ, Moore TD, Lele SS, et al. Diastolic ventricular interaction in chronic heart failure. Lancet 1997;349:1720-4.

14 Janicki JS, Weber KT. The pericardium and ventricular interaction, distensibility, and function. Am J Physiol 1980;238:H494-503.

15 Baker BJ, Wilen MM, Boyd CM, et al. Relation of right ventricular ejection fraction to exercise capacity in chronic left ventricular failure. Am J Cardiol 1984; 54:596-9.

16 Atherton JJ, Moore TD, Thomson HL, et al. Restrictive left ventricular filling patterns are predictive of diastolic ventricular interaction in chronic heart failure. J Am Coll Cardiol 1998;31:413-8.

17 Giannuzzi P, Imparato A, Temporelli PL, et al. Doppler-derived mitral deceleration time of early filling as a strong predictor of pulmonary capillary wedge pressure in postinfarction patients with left ventricular systolic dysfunction. J Am Coll Cardiol 1994;23:1630-7.

18 Atherton JJ, Thomson HL, Moore TD, et al. Diastolic ventricular interaction: a possible mechanism for abnormal vascular responses during volume unloading in heart failure. Circulation 1997;96:4273-9.

19 Atherton JJ, Blackman DJ, Moore TD, et al. Diastolic ventricular interaction in chronic heart failure: relation to heart rate variability and neurohumoral status. Heart Vessels 1998;13:269-77.

20 Francis GS, Cohn JN, Johnson G, et al. Plasma norepinephrine, plasma renin activity, and congestive heart failure. Relations to survival and the effects of therapy in V-HeFT II. The V-HeFT VA cooperative studies group. Circulation 1993;87:V|40-|48.

21 Osterziel KJ, Hanlein D, Willenbrock R, et al. Baroreflex sensitivity and cardiovascular mortality in patients with mild to moderate heart failure. $\mathrm{Br}$ Heart J 1995:73:517-22.

22 lonescu AA, lonescu AA, Payne N, et al. Subclinical right ventricular dysfunction in cystic fibrosis. A study using Doppler echocardiography. Am J Respir Crit Care Med 2001;163:1212-8.

23 Kukulski T, Hubbert L, Arnold $M$, et al. Normal regional right ventricular function and its change with age: a Doppler myocardial imaging study. J Am Soc Echocardiogr 2000;13:194-204. 